\title{
Need To Treat Lymphatic Malformations
}

Indrani $\mathbf{S}^{*}$, Stephen E, Selvaraj D, Premkumar P and Agarwal S

Department of Vascular Surgery, Christian Medical College, Vellore, Tamil Nadu, India

*Corresponding author: Indrani Sen, Department of Vascular Surgery, Christian Medical College, Vellore, 632004, Tamil nadu, India, Tel: 091 04162282085; Fax: 091 04162282035; E-mail: dr.indranisen@gmail.com

Rec date: Jan 17, 2014, Acc date: June 03, 2014, Pub date: June 05, 2014

Copyright: ( 2014 Indrani S, et al. This is an open-access article distributed under the terms of the Creative Commons Attribution License, which permits unrestricted use, distribution, and reproduction in any medium, provided the original author and source are credited.

\begin{abstract}
Both truncular and extratruncular forms of lymphatic malformations; as described in the Hamburg classification; remain difficult to treat. Most of these malformations are treated adequately or present with complications (recurrent infection, cellulitis, bleeding, skin ooze) increasing the therapeutic challenge. We present a case with complications of a LM highlighting the need for early intervention for these lesions. A 19 year old male presented with watery discharge from the skin over a left thigh swelling since childhood. He was diagnosed to have an extratruncular Lymphatic Malformation (LM). Treatment was deferred. He presented with necrosis of the skin and subcutaneous tissue after 3 months requiring debridement and split thickness skin graft.
\end{abstract}

\section{Case History}

A 19 year old boy had presented with a left thigh swelling with intermittent serous discharge from the skin since childhood. He had episodes of fever with redness and pain of the involved area suggestive of infection. MR imaging revealed multiple cystic spaces - duplex revealed minimal flow, a micro and macrocystic lymphatic malformation of the left leg extending into the pelvis (Figure 1) was diagnosed. He was advised conservative management.
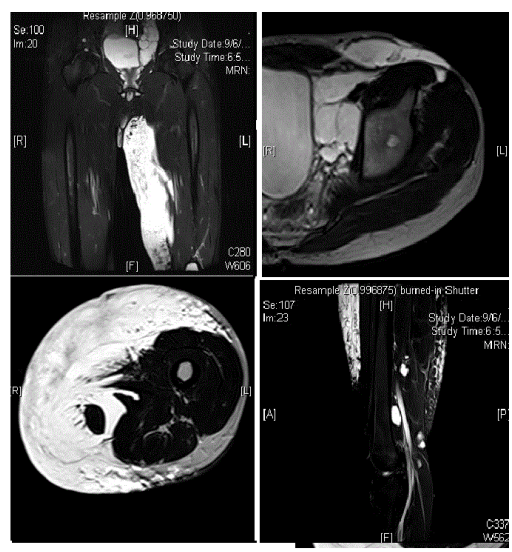

Imaging

He presented 3 months later with necrosis of the skin and subcutaneous tissue. On examination, the left thigh was involved nearly circumferentially with an eschar (Figure 2).

Peripheral pulses were palpable and the calf was supple. He underwent debridement of the left thigh eschar, skin and subcutaneous tissue. Infected lymphatic fluid in the macrocystic lesions in the inguinal area was drained. Blood counts ruled out lymphocytopenia.

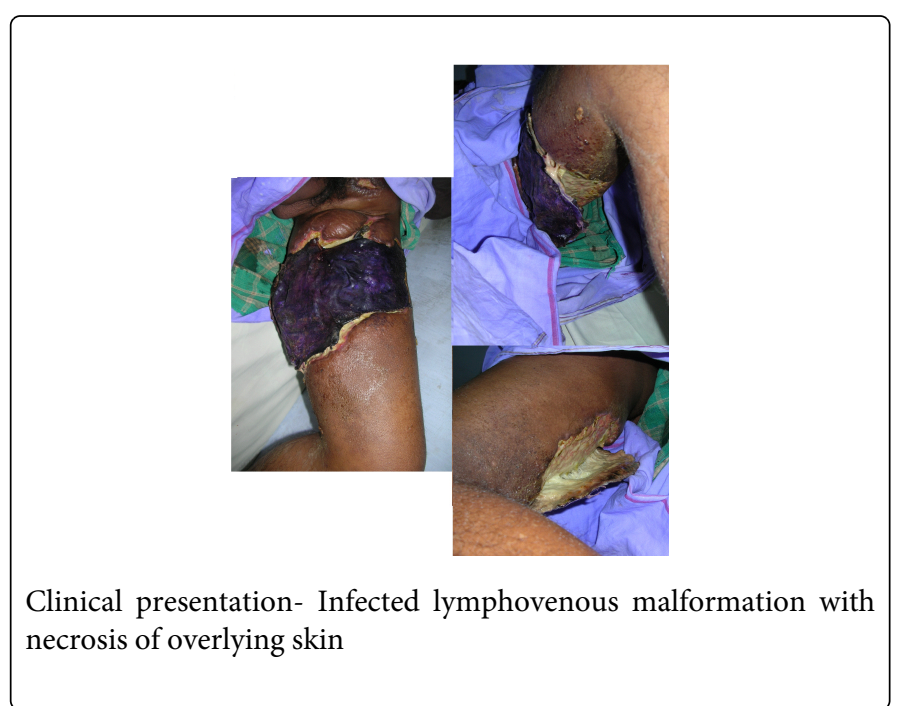

The culture revealed streptococcus sensitive to penicillin- patient was treated with a week of culture specific antibiotics. The wound was managed with dressings and a split thickness skin graft was applied once the granulation tissue was healthy. Graft take was good. The intermittent discharge from the surrounding skin persisted and the grafted skin also developed this at 18 month follow up (Figure 3).

He was continued on compression garments, manual lymphatic drainage (complex decongestive therapy) and repeated tetracycline sclerotherapy for the remnant lesions and put on oral antibiotic (penicillin) prophylaxis with which the number and severity of the infections of the infections have reduced (one in 3 years). 


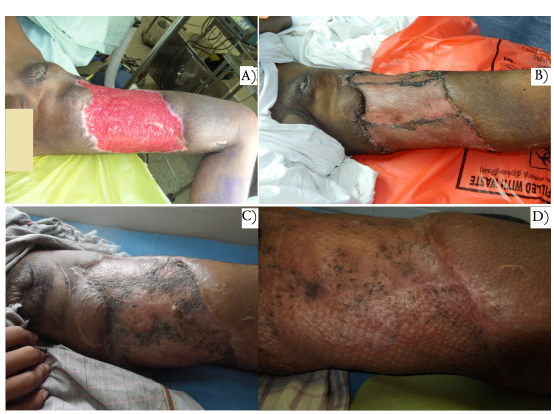

Follow up A) Immediate post debridement; B) Skin graft postoperative day 7; C) 12 months post operation; D) 18 months post operation

\section{Discussion}

Our patient was diagnosed to have a LM only in adulthood highlighting the need for increased awareness of the nature of disease and the treatment options. In our patient, the necrosis probably started from the lymphatic component, due to infection. Early elective treatment may have averted the episode of necrosis with severe sepsis. Even when presenting with complications, treatment with culture specific antibiotics, debridement and secondary cover can achieve an acceptable clinical outcome. Antibiotic prophylaxis with oral penicillin has helped in reducing the frequency of secondary infection. Though Lymphatic Malformations (LM) are not intrinsically life or limb threatening, they may become so when complications, such as lymph leakage or recurrent infections occur and hence should be treated before these set in. In combined LVM, an episode of thrombosis may be the initial triggering event which can then lead on to other local or systemic complications.

Lymphatic and venolymphatic malformations may be localized or diffuse and can affect any part of the body [1,2]. These lesions are difficult to treat and are sometimes left untreated till they present with complications. The exact pathogenesis of the lesions is not well understood. These may involve contiguous or distinct areas; associations with gene mutations- both sporadic and familial are known [3].

There are two main systems of classification. The Hamburg classification classifies lesions into truncular and extratruncular forms: incorporating the embryological origin, morphological differences, unique characteristics, prognosis and recurrence rates of these lesions [4]. The ISSVA classification considers the clinical history, disease course, and treatment options. It divides vascular anomalies into vasoproliferative neoplasms or vascular malformations; malformations only grow in proportion to the growth of the child as the cells in these lesions do not have increased endothelial cell mitosis. Both these systems have clinical applicability and are used in conjunction in management of complex lesions.

The treatment of lymphatic malformations is guided by functional / cosmetic disability. Lesions located near vital organs, and those that threaten vital functions, or cause complications (recurrent infection/ intralesional bleeding) also warrant early intervention $[5,6]$. Surgical excision and injection sclerotherapy (bleomycin, tertracycline, OK 432, ethanol) are used in conjunction with compressive garments and complex decongestive therapy [4,7]. CO2, YAG and KTP lasers have been used to treat skin vesicles [8]. Complex debulking/ reconstructive surgery is offered in specialized centers [4]. Patients may have alterations of the immune system with poor immunity secondary to lymphocytopenia- long term or peri-procedural antibiotics prophylaxis may be required [9]. Antibiotic prophylaxis helps in reducing the frequency of infection. However, patients must be counseled about the nature of the disease, instructed about how to identify early signs of infection and seek medical care early.

Newer antiangiogenic agents are under study; at present a multidisciplinary team approach consisting of vascular surgeons, interventional radiologists; physiotherapists, plastic and reconstructive surgery is best in planning treatment for these patients $[1,10]$.

\section{Acknowledgement}

We would like to thank Dr. BB Lee for the time and input in helping advance our understanding of the treatment of vascular malformations and in preparation of this manuscript.

\section{References}

1. Blatt J, McLean TW, Castellino SM, Burkhart CN (2013) A review of contemporary options for medical management of hemangiomas, other vascular tumors, and vascular malformations. Pharmacol. Ther 139: 327333.

2. Lee BB, Laredo J, Lee SJ, Huh SH, Joe JH, et al. (2007) Congenital vascular malformations: general diagnostic principles. Phlebology 22: 253-257.

3. Burrows PE, Gonzalez-Garay ML, Rasmussen JC, Aldrich MB, Guilliod $\mathrm{R}$, et al. (2013) Lymphatic abnormalities are associated with RASA1 gene mutations in mouse and man. Proc Natl Acad Sci U S A 110: 8621-8626.

4. Lee BB, Kim YW, Seo JM, Hwang JH, Do YS, et al. (2005) Current concepts in lymphatic malformation. Vasc Endovascular Surg 39: 67-81.

5. López López AJ, Gómez Farpón A, Vega Mata N, Montalvo Avalos C, Oviedo Gutiérrez M, et al. (2013) [Abdominal lymphatic malformation (ALM). Our experience]. Cir Pediatr 26: 17-20.

6. Thottam PJ, Al-Barazi R, Madgy DN, Rozzelle A (2013) Submucosal resection of a microcystic oropharyngeal lymphatic malformation using radiofrequency ablation. Int. J. Pediatr. Otorhinolaryngol 77: 1589-1592

7. Fraulin FO, Flannigan RK, Sharma VK, McPhalen DF, Harrop RA (2012) The epidemiological profile of the Vascular Birthmark Clinic at the Alberta Children's Hospital. Can J Plast Surg 20: 67-70.

8. Savas JA, Ledon J, Franca K, Chacon A, Zaiac M, et al. (2013) Carbon dioxide laser for the treatment of microcystic lymphatic malformations (lymphangioma circumscriptum): a systematic review. Dermatol Surg 39: 1147-1157.

9. Ernemann U, Hoffmann J, Breuninger H, Reinert S, Skalej M (2002) [Interdisciplinary concept for classification and treatment of vascular anomalies in the head and neck]. Mund Kiefer Gesichtschir 6: 402-409.

10. Tempero RM, Hannibal M, Finn LS, Manning SC, Cunningham ML, et al. (2006) Lymphocytopenia in children with lymphatic malformation. Arch Otolaryngol Head Neck Surg 132: 93-97. 